\title{
AN EXAMPLE OF SPECTRAL PHASE TRANSITION PHENOMENON IN A CLASS OF JACOBI MATRICES WITH PERIODICALLY MODULATED WEIGHTS
}

\author{
SERGEY SIMONOV
}

\begin{abstract}
We consider self-adjoint unbounded Jacobi matrices with diagonal $q_{n}=n$ and weights $\lambda_{n}=c_{n} n$, where $c_{n}$ is a 2-periodical sequence of real numbers. The parameter space is decomposed into several separate regions, where the spectrum is either purely absolutely continuous or discrete. This constitutes an example of the spectral phase transition of the first order. We study the lines where the spectral phase transition occurs, obtaining the following main result: either the interval $\left(-\infty ; \frac{1}{2}\right)$ or the interval $\left(\frac{1}{2} ;+\infty\right)$ is covered by the absolutely continuous spectrum, the remainder of the spectrum being pure point. The proof is based on finding asymptotics of generalized eigenvectors via the Birkhoff-Adams Theorem. We also consider the degenerate case, which constitutes yet another example of the spectral phase transition.
\end{abstract}

\section{INTRODUCTION}

In the present paper we study a class of Jacobi matrices with unbounded entries: a linearly growing diagonal and periodically modulated linearly growing weights.

We first define the operator $J$ on the linear set of vectors $l_{\text {fin }}(\mathbb{N})$ having finite number of non-zero elements:

$$
(J u)_{n}=\lambda_{n-1} u_{n-1}+q_{n} u_{n}+\lambda_{n} u_{n+1}, n \geq 2
$$

with the initial condition $(J u)_{1}=q_{1} u_{1}+\lambda_{1} u_{2}$, where $q_{n}=n, \lambda_{n}=c_{n} n$, and $c_{n}$ is a real 2-periodic sequence, generated by the parameters $c_{1}$ and $c_{2}$.

Let $\left\{e_{n}\right\}_{n \in \mathbb{N}}$ be the canonical basis in $l^{2}(\mathbb{N})$. With respect to this basis the operator $J$ admits the following matrix representation:

$$
J=\left(\begin{array}{cccc}
q_{1} & \lambda_{1} & 0 & \ldots \\
\lambda_{1} & q_{2} & \lambda_{2} & \ldots \\
0 & \lambda_{2} & q_{3} & \ldots \\
\vdots & \vdots & \vdots & \ddots
\end{array}\right)
$$

Due to the Carleman condition [2] $\sum_{n=1}^{\infty} \frac{1}{\lambda_{n}}=\infty$, the operator $J$ is essentially self-adjoint. We will therefore assume throughout the paper, that $J$ is a closed self-adjoint operator in $l^{2}(\mathbb{N})$, defined on its natural domain $D(J)=\left\{u \in l^{2}(\mathbb{N})\right.$ : $\left.J u \in l^{2}(\mathbb{N})\right\}$.

We base our spectrum investigation on the subordinacy theory due to Gilbert and Pearson [6], generalized to the case of Jacobi matrices by Khan and Pearson

1991 Mathematics Subject Classification. 47A10, 47B36.

Key words and phrases. Jacobi matrices, Spectral phase transition, Absolutely continuous spectrum, Pure point spectrum, Discrete spectrum, Subordinacy theory, Asymptotics of generalized eigenvectors. 
12. Using this theory, we study an example of spectral phase transition of the first order. This example was first obtained by Naboko and Janas in [9] and [10]. In cited articles, the authors managed to demonstrate that the space of parameters $\left(c_{1} ; c_{2}\right) \in \mathbb{R}^{2}$ can be naturally decomposed into a set of regions of two types. In the regions of the first type, the spectrum of the operator $J$ is purely absolutely continuous and covers the real line $\mathbb{R}$, whereas in the regions of the second type the spectrum is discrete.

Due to [9] and [10, spectral properties of Jacobi matrices of our class are determined by the location of the point zero relative to the absolutely continuous spectrum of a certain periodic matrix $J_{\text {per }}$, constructed based on the modulation parameters $c_{1}$ and $c_{2}$. In our case this leads to:

$$
J_{p e r}=\left(\begin{array}{ccccc}
1 & c_{1} & 0 & 0 & \ldots \\
c_{1} & 1 & c_{2} & 0 & \ldots \\
0 & c_{2} & 1 & c_{1} & \ldots \\
0 & 0 & c_{1} & 1 & \ldots \\
\vdots & \vdots & \vdots & \vdots & \ddots
\end{array}\right)
$$

Considering the characteristic polynomial

$$
d_{J_{p e r}}(\lambda)=\operatorname{Tr}\left(\left(\begin{array}{cc}
0 & 1 \\
-\frac{c_{1}}{c_{2}} & \frac{\lambda-1}{c_{2}}
\end{array}\right)\left(\begin{array}{cc}
0 & 1 \\
-\frac{c_{2}}{c_{1}} & \frac{\lambda-1}{c_{1}}
\end{array}\right)\right)=\frac{(\lambda-1)^{2}-c_{1}^{2}-c_{2}^{2}}{c_{1} c_{2}},
$$

the location of the absolutely continuous spectrum $\sigma_{a c}\left(J_{p e r}\right)$ of $J_{p e r}$ can then be determined from the following condition [2]:

$$
\lambda \in \sigma_{a c}\left(J_{p e r}\right) \Leftrightarrow\left|d_{J_{p e r}}(\lambda)\right| \leq 2 .
$$

This leads to the following result [10, concerning the spectral structure of the operator $J$.

If $\left|d_{J_{p e r}}(0)\right|<2$, then the spectrum of the operator $J$ is purely absolutely continuous, covering the whole real line.

If, on the other hand, $\left|d_{J_{p e r}}(0)\right|>2$, then the spectrum of the operator $J$ is discrete.

Thus, the condition $\left|\frac{1-c_{1}^{2}-c_{2}^{2}}{c_{1} c_{2}}\right|=2$, equivalent to $\left\{\left|c_{1}\right|+\left|c_{2}\right|=1\right.$ or ||$c_{1}|-| c_{2}||=$ $1\}$, determines the boundaries of the above mentioned regions on the plane $\left(c_{1} ; c_{2}\right)$ where one of the cases holds and the spectrum of the operator $J$ is either purely absolutely continuous or discrete (see fig. 1 on page 8).

Note also, that Jacobi matrices with modulation parameters equal to $\pm c_{1}$ and $\pm c_{2}$ are unitarily equivalent. Thus the situation can be reduced to studying the case $c_{1}, c_{2}>0$.

In the present paper we attempt to study the spectral structure on the lines, where the spectral phase transition occurs, i.e., on the lines separating the aforementioned regions.

The paper is organized as follows.

Section 2 deals with the calculation of the asymptotics of generalized eigenvectors of the operator $J$. This calculation is mainly based on the Birkhoff-Adams Theorem 4. The asymptotics are then used to characterize the spectral structure of the operator via the Khan-Pearson Theorem [12. It turns out, that on the lines 
where the spectral phase transition occurs the spectrum is neither purely absolutely continuous nor pure point, but a combination of both.

In Section 3, we attempt to ascertain whether the pure point part of the spectrum is actually discrete. In doing so, we establish a criterion that guarantees that the operator $J$ is semibounded from below, for all $\left(c_{1} ; c_{2}\right) \in \mathbb{R}^{2}$. This semiboundedness is then used in conjunction with classical methods of operator theory to prove, that in at least one situation the discreteness of the pure point spectrum is guaranteed.

Section 4 is dedicated to the study of the degenerate case, i.e., the case when one of the modulation parameters turns to zero. In this situation, one can explicitly calculate all eigenvalues of the operator. On this route we obtain yet another "hidden" example of the spectral phase transition of the first order as the point $\left(c_{1} ; c_{2}\right)$ moves along one of the critical lines in the space of parameters.

\section{Generalized eigenvectors and the spectrum of the operator J}

In this Section, we calculate asymptotics of generalized eigenvectors of the operator $J$. Consider the recurrence relation [12]

$$
\lambda_{n-1} u_{n-1}+\left(q_{n}-\lambda\right) u_{n}+\lambda_{n} u_{n+1}=0, n \geq 2 .
$$

We reduce it to a form such that the Birkhoff-Adams Theorem is applicable. To this end, we need to have a recurrence relation of the form:

$$
x_{n+2}+F_{1}(n) x_{n+1}+F_{2}(n) x_{n}=0, n \geq 1,
$$

where $F_{1}(n)$ and $F_{2}(n)$ admit the following asymptotical expansions as $n \rightarrow \infty$ :

$$
F_{1}(n) \sim \sum_{k=0}^{\infty} \frac{a_{k}}{n^{k}}, F_{2}(n) \sim \sum_{k=0}^{\infty} \frac{b_{k}}{n^{k}}
$$

with $b_{0} \neq 0$. Consider the characteristic equation $\alpha^{2}+a_{0} \alpha+b_{0}=0$ and denote its roots $\alpha_{1}$ and $\alpha_{2}$. Then [4]:

Theorem (Birkhoff-Adams). There exist two linearly independent solutions $x_{n}^{(1)}$ and $x_{n}^{(2)}$ of the recurrence relation (2.2) with the following asymptotics as $n \rightarrow \infty$ :

1 .

$$
x_{n}^{(i)}=\alpha_{i}^{n} n^{\beta_{i}}\left(1+O\left(\frac{1}{n}\right)\right), i=1,2,
$$

if the roots $\alpha_{1}$ and $\alpha_{2}$ are different, where $\beta_{i}=\frac{a_{1} \alpha_{i}+b_{1}}{a_{0} \alpha_{i}+2 b_{0}}, \quad i=1,2$.

2.

$$
x_{n}^{(i)}=\alpha^{n} e^{\delta_{i} \sqrt{n}} n^{\beta}\left(1+O\left(\frac{1}{\sqrt{n}}\right)\right), i=1,2,
$$

if the roots $\alpha_{1}$ and $\alpha_{2}$ coincide, $\alpha:=\alpha_{1}=\alpha_{2}$, and an additional condition $a_{1} \alpha+$ $b_{1} \neq 0$ holds, where $\beta=\frac{1}{4}+\frac{b_{1}}{2 b_{0}}, \delta_{1}=2 \sqrt{\frac{a_{0} a_{1}-2 b_{1}}{2 b_{0}}}=-\delta_{2}$.

This Theorem is obviously not directly applicable in our case, due to wrong asymptotics of coefficients at infinity. In order to deal with this problem, we study a pair of recurrence relations, equivalent to (2.1), separating odd and even components of a vector $u$. This allows us to apply the Birkhoff-Adams Theorem to each of the recurrence relations of the pair, which yields the corresponding asymptotics. Combining the two asymptotics together, we then obtain the desired result for the solution of (2.1). 
Denoting $v_{k}:=u_{2 k-1}$ and $w_{k}:=u_{2 k}$, we rewrite the recurrence relation (2.1) for the consecutive values of $n: n=2 k$ and $n=2 k+1$.

$$
\begin{gathered}
\lambda_{2 k-1} v_{k}+\left(q_{2 k}-\lambda\right) w_{k}+\lambda_{2 k} v_{k+1}=0, \\
\lambda_{2 k} w_{k}+\left(q_{2 k+1}-\lambda\right) v_{k+1}+\lambda_{2 k+1} w_{k+1}=0 .
\end{gathered}
$$

Then we exclude $w$ in order to obtain the recurrence relation for $v$ :

$$
\begin{gathered}
w_{k}=-\frac{\lambda_{2 k-1} v_{k}+\lambda_{2 k} v_{k+1}}{q_{2 k}-\lambda}, \\
v_{k+2}+P_{1}(k) v_{k+1}+P_{2}(k) v_{k}=0, k \geq 1,
\end{gathered}
$$

where

$$
\begin{gathered}
P_{1}(k)=\frac{q_{2 k+2}-\lambda}{q_{2 k}-\lambda} \frac{\lambda_{2 k}^{2}}{\lambda_{2 k+1} \lambda_{2 k+2}}-\frac{\left(q_{2 k+1}-\lambda\right)\left(q_{2 k+2}-\lambda\right)}{\lambda_{2 k+1} \lambda_{2 k+2}}+\frac{\lambda_{2 k+1}}{\lambda_{2 k+2}}, \\
P_{2}(k)=\frac{q_{2 k+2}-\lambda}{q_{2 k}-\lambda} \frac{\lambda_{2 k-1} \lambda_{2 k}}{\lambda_{2 k+1} \lambda_{2 k+2}},
\end{gathered}
$$

In our case $\left(\lambda_{n}=c_{n} n\right.$ and $\left.q_{n}=n\right)$ this yields the following asymptotic expansions (cf. (2.3) ) for $P_{1}(k)$ and $P_{2}(k)$ as $k$ tends to infinity:

$$
P_{1}(k)=\sum_{j=0}^{\infty} \frac{a_{j}}{k^{j}}, P_{2}(k)=\sum_{j=0}^{\infty} \frac{b_{j}}{k^{j}}
$$

with

$$
\begin{gathered}
a_{0}=\frac{c_{1}^{2}+c_{2}^{2}-1}{c_{1} c_{2}}, a_{1}=-\frac{c_{1}^{2}+c_{2}^{2}-2 \lambda}{2 c_{1} c_{2}}=-\frac{a_{0}}{2}+\frac{\lambda-\frac{1}{2}}{c_{1} c_{2}}, \\
b_{0}=1, b_{1}=-1 .
\end{gathered}
$$

The remaining coefficients $\left\{a_{j}\right\}_{j=2}^{+\infty},\left\{b_{j}\right\}_{j=2}^{+\infty}$ can also be calculated explicitly.

On the same route one can obtain the recurrence relation for even components $w_{k}$ of the vector $u$ :

$$
w_{k+2}+R_{1}(k) w_{k+1}+R_{2}(k) w_{k}=0, k \geq 1 .
$$

Note, that if $k$ is substituted in (2.4) by $k+\frac{1}{2}$ and $v$ by $w$, the equation (2.4) turns into (2.6). Therefore,

$$
R_{1}(k)=P_{1}\left(k+\frac{1}{2}\right), R_{2}(k)=P_{2}\left(k+\frac{1}{2}\right),
$$

and thus as $k \rightarrow \infty$,

$$
\begin{aligned}
& R_{1}(k)=a_{0}+\frac{a_{1}}{k}+O\left(\frac{1}{k^{2}}\right), \\
& R_{2}(k)=b_{0}+\frac{b_{1}}{k}+O\left(\frac{1}{k^{2}}\right),
\end{aligned}
$$

with $a_{0}, a_{1}, b_{0}, b_{1}$ defined by (2.5).

Applying now the Birkhoff-Adams Theorem we find the asymptotics of solutions of recurrence relations (2.4) and (2.6). This leads to the following result. 
Lemma 2.1. Recurrence relations (2.4) and (2.6) have solutions $v_{n}^{+}, v_{n}^{-}$and $w_{n}^{+}$, $w_{n}^{-}$, respectively, with the following asymptotics as $k \rightarrow \infty$ :

1.

$$
v_{k}^{ \pm}, w_{k}^{ \pm}=\alpha_{ \pm}^{k} k^{\beta_{ \pm}}\left(1+O\left(\frac{1}{k}\right)\right)
$$

if $\left|\frac{c_{1}^{2}+c_{2}^{2}-1}{c_{1} c_{2}}\right| \neq 2$, where $\alpha_{+}$and $\alpha_{-}$are the roots of the equation $\alpha^{2}+a_{0} \alpha+b_{0}=0$ and $\beta_{ \pm}=\frac{a_{1} \alpha_{ \pm}+b_{1}}{a_{0} \alpha_{ \pm}+2 b_{0}}$ with $a_{0}, a_{1}, b_{0}, b_{1}$ defined by (2.5).

Moreover, if $\left|\frac{c_{1}^{2}+c_{2}^{2}-1}{c_{1} c_{2}}\right|>2$ then $\alpha_{ \pm}$are real and $\left|\alpha_{-}\right|<1<\left|\alpha_{+}\right|$,

whereas if $\left|\frac{c_{1}^{2}+c_{2}^{2}-1}{c_{1} c_{2}}\right|<2$ then $\alpha_{+}=\overline{\alpha_{-}}, \beta_{+}=\overline{\beta_{-}}$and the vectors $v^{+}, v^{-}, w^{+}, w^{-}$ are not in $l^{2}(\mathbb{N})$.

2.

$$
v_{k}^{ \pm}, w_{k}^{ \pm}=\alpha^{k} k^{-\frac{1}{4}} e^{\delta_{ \pm} \sqrt{k}}\left(1+O\left(\frac{1}{\sqrt{k}}\right)\right)
$$

if $\left|\frac{c_{1}^{2}+c_{2}^{2}-1}{c_{1} c_{2}}\right|=2$ and $\lambda \neq \frac{1}{2}$, where $\alpha=\alpha_{+}=\alpha_{-}$.

Moreover, if $\frac{c_{1}^{2}+c_{2}^{2}-1}{c_{1} c_{2}}=2$, then $\delta_{+}=2 \sqrt{\frac{2 \lambda-1}{2 c_{1} c_{2}}}=-\delta_{-}$, whereas if $\frac{c_{1}^{2}+c_{2}^{2}-1}{c_{1} c_{2}}=-2$, then $\delta_{+}=2 \sqrt{\frac{1-2 \lambda}{2 c_{1} c_{2}}}=-\delta_{-}$.

Proof. Consider recurrence relation (2.4) and let the constants $a_{0}, a_{1}, b_{0}, b_{1}$ be defined by (2.5). Consider the characteristic equation $\alpha^{2}+a_{0} \alpha+b_{0}=0$. It has different roots, $\alpha_{-}<\alpha_{+}$, when the discriminant $D$ differs from zero: $D=$ $\left(\frac{c_{1}^{2}+c_{2}^{2}-1}{c_{1} c_{2}}\right)^{2}-4 \neq 0$. Note that $\alpha_{+} \alpha_{-}=1$.

Consider the case $D<0$. A direct application of the Birkhoff-Adams Theorem yields:

$$
v_{k}^{ \pm}=\alpha_{ \pm}^{k} k^{\beta_{ \pm}}\left(1+O\left(\frac{1}{k}\right)\right), k \rightarrow \infty
$$

where $\beta_{ \pm}=\frac{a_{1} \alpha_{ \pm}+b_{1}}{a_{0} \alpha \pm+2 b_{0}}$. Then $\alpha_{+}=\overline{\alpha_{-}},\left|\alpha_{+}\right|=\left|\alpha_{-}\right|=1$ and $\beta_{+}=\overline{\beta_{-}}$. Note also, that $v^{ \pm}$are not in $l^{2}$ :

$$
\operatorname{Re} \beta_{+}=\operatorname{Re} \beta_{-}=-\frac{1}{2}+\frac{2 \lambda-1}{2 c_{1} c_{2}} \operatorname{Re}\left(\frac{1}{a_{0}+2 \alpha_{-}}\right)=-\frac{1}{2} .
$$

In the case $D>0, \alpha_{+}$and $\alpha_{-}$are real and $\left|\alpha_{-}\right|<1<\left|\alpha_{+}\right|$, hence $v^{-}$lies in $l^{2}$.

Ultimately, in the case $D=0$, the roots of the characteristic equation coincide and are equal to $\alpha=-\frac{a_{0}}{2}$, with $|\alpha|=1$, and the additional condition $a_{0} a_{1} \neq 2 b_{1}$ is equivalent to

$$
-\frac{a_{0}^{2}}{2}+\frac{a_{0}\left(\lambda-\frac{1}{2}\right)}{c_{1} c_{2}} \neq-2 \Leftrightarrow \lambda \neq \frac{1}{2} .
$$

The Birkhoff-Adams Theorem yields:

$$
v_{k}^{ \pm}=\alpha^{k} k^{\beta} e^{\delta_{ \pm} \sqrt{k}}\left(1+O\left(\frac{1}{\sqrt{k}}\right)\right), k \rightarrow \infty,
$$

where $\beta=-\frac{1}{4}, \delta_{+}=2 \sqrt{\frac{a_{0}\left(\lambda-\frac{1}{2}\right)}{2 c_{1} c_{2}}}=-\delta_{-}$. If the value $\delta_{+}$is pure imaginary, then clearly the vectors $v^{ \pm}$do not belong to $l^{2}(\mathbb{N})$. 
In order to prove the assertion of the Lemma in relation to $w^{ \pm}$, note that in our calculations we use only the first two orders of the asymptotical expansions for $P_{1}(k)$ and $P_{2}(k)$. These coincide with the ones for $R_{1}(k)$ and $R_{2}(k)$. Thus, the solutions of recurrence relations (2.4) and (2.6) coincide in their main orders, which completes the proof.

Now we are able to solve the recurrence relation (2.1) combining the solutions of recurrence relations (2.4) and (2.6).

Lemma 2.2. Recurrence relation (2.1) has two linearly independent solutions $u_{n}^{+}$ and $u_{n}^{-}$with the following asymptotics as $k \rightarrow \infty$ :

1.

$$
\left\{\begin{array}{l}
u_{2 k-1}^{ \pm}=\alpha_{ \pm}^{k} k^{\beta_{ \pm}}\left(1+O\left(\frac{1}{k}\right)\right) \\
u_{2 k}^{ \pm}=-\left(c_{1}+\alpha_{ \pm} c_{2}\right) \alpha_{ \pm}^{k} k^{\beta_{ \pm}}\left(1+O\left(\frac{1}{k}\right)\right)
\end{array}\right.
$$

if $\left|\frac{c_{1}^{2}+c_{2}^{2}-1}{c_{1} c_{2}}\right| \neq 2$, where the values $\alpha_{+}, \alpha_{-}, \beta_{+}, \beta_{-}$are taken from the statement of Lemma 2.1

2.

$$
\left\{\begin{array}{l}
u_{2 k-1}^{ \pm}=\alpha^{k} e^{\delta_{ \pm} \sqrt{k}} k^{-\frac{1}{4}}\left(1+O\left(\frac{1}{\sqrt{k}}\right)\right) \\
u_{2 k}^{ \pm}=-\left(c_{1}+\alpha c_{2}\right) \alpha^{k} e^{\delta_{ \pm} \sqrt{k}} k^{-\frac{1}{4}}\left(1+O\left(\frac{1}{\sqrt{k}}\right)\right)
\end{array}\right.
$$

if $\left|\frac{c_{1}^{2}+c_{2}^{2}-1}{c_{1} c_{2}}\right|=2$ and $\lambda \neq \frac{1}{2}$, where the values $\alpha, \delta_{+}, \delta_{-}$are taken from the statement of Lemma 2.1.

Proof. It is clear, that any solution of recurrence relation 2.1) $u$ gives two vectors, $v$ and $w$, constructed of its odd and even components, which solve recurrence relations (2.4) and (2.6), respectively. Consequently, any solution of the recurrence relation (2.1) belongs to the linear space with the basis $\left\{V^{+}, V^{-}, W^{+}, W^{-}\right\}$, where

$$
V_{2 k-1}^{ \pm}=v_{k}^{ \pm}, V_{2 k}^{ \pm}=0 \text { and } W_{2 k-1}^{ \pm}=0, W_{2 k}^{ \pm}=w_{k}^{ \pm} .
$$

This 4-dimensional linear space contains 2-dimensional subspace of solutions of recurrence relation (2.1). In order to obtain a solution $u$ of (2.1), one has to obtain two conditions on the coefficients $a_{+}, a_{-}, b_{+}, b_{-}$such that $u=a_{+} V^{+}+a_{-} V^{-}+$ $b_{+} W^{+}+b_{-} W^{-}$,

$$
u_{2 k-1}=a_{+} v_{k}^{+}+a_{-} v_{k}^{-}, u_{2 k}=b_{+} w_{k}^{+}+b_{-} w_{k}^{-} .
$$

Using Lemma 2.1. we substitute the asymptotics of this $u$ into (2.1) where $n$ is taken equal to $2 k$,

$$
\lambda_{2 k-1} u_{2 k-1}+\left(q_{2 k}-\lambda\right) u_{2 k}+\lambda_{2 k} u_{2 k+1}=0 .
$$

As in Lemma 2.1, we have two distinct cases.

Consider the case $\left|\frac{c_{1}^{2}+c_{2}^{2}-1}{c_{1} c_{2}}\right| \neq 2$. Then

$$
\begin{gathered}
\left(c_{1}\left[a_{+}\left(\frac{\alpha_{+}}{\alpha_{-}}\right)^{k} k^{\left(\beta_{+}-\beta-\right)}+a_{-}\right]+\left[b_{+}\left(\frac{\alpha_{+}}{\alpha_{-}}\right)^{k} k^{\left(\beta_{+}-\beta-\right)}+b_{-}\right]+\right. \\
\left.+c_{2}\left[a_{+}\left(\frac{\alpha_{+}}{\alpha_{-}}\right)^{k} \alpha_{+} k^{\left(\beta_{+}-\beta-\right)}+a_{-} \alpha_{-}\right]\right)(1+o(1))=0 \text { as } k \rightarrow \infty .
\end{gathered}
$$


Therefore, for any number $k$ greater than some big enough positive $K$ one has:

$$
\left[c_{1} a_{+}+b_{+}+c_{2} a_{+} \alpha_{+}\right]\left(\frac{\alpha_{+}}{\alpha_{-}}\right)^{k} k^{\left(\beta_{+} \beta_{-}\right)}+\left[c_{1} a_{-}+b_{-}+c_{2} a_{-} \alpha_{-}\right]=0,
$$

hence $b_{ \pm}=-\left(c_{1}+\alpha_{ \pm} c_{2}\right) a_{ \pm}$. Thus (2.9) admits the following form:

$$
\begin{gathered}
u_{2 k-1}=a_{+} v_{k}^{+}+a_{-} v_{k}^{-} \\
u_{2 k}=-\left(c_{1}+\alpha_{+} c_{2}\right) a_{+} w_{k}^{+}-\left(c_{1}+\alpha_{-} c_{2}\right) a_{-} w_{k}^{-} .
\end{gathered}
$$

It is clear now, that the vectors $u^{+}$and $u^{-}$defined as follows:

$$
\begin{aligned}
& u_{2 k-1}^{+}=v_{k}^{+}, u_{2 k}^{+}=-\left(c_{1}+\alpha_{+} c_{2}\right) w_{k}^{+}, \\
& u_{2 k-1}^{-}=v_{k}^{-}, u_{2 k}^{-}=-\left(c_{1}+\alpha_{-} c_{2}\right) w_{k}^{-},
\end{aligned}
$$

are two linearly independent solutions of the recurrence relation (2.1).

The second case here, $\left|\frac{c_{1}^{2}+c_{2}^{2}-1}{c_{1} c_{2}}\right|=2$, can be treated in an absolutely analogous fashion.

Due to Gilbert-Pearson-Khan subordinacy theory [6], [12, we are now ready to prove our main result concerning the spectral structure of the operator $J$.

Theorem 2.3. Depending on the modulation parameters $c_{1}$ and $c_{2}$, there are four distinct cases, describing the spectral structure of the operator $J$ :

(a) If $\left|\frac{c_{1}^{2}+c_{2}^{2}-1}{c_{1} c_{2}}\right|<2$, the spectrum is purely absolutely continuous with local multiplicity one almost everywhere on $\mathbb{R}$,

(b) If ||$c_{1}|-| c_{2}||=1$ and $c_{1} c_{2} \neq 0$, the spectrum is purely absolutely continuous with local multiplicity one almost everywhere on $\left(-\infty ; \frac{1}{2}\right)$ and pure point on $\left(\frac{1}{2} ;+\infty\right)$,

(c) If $\left|c_{1}\right|+\left|c_{2}\right|=1$ and $c_{1} c_{2} \neq 0$, the spectrum is purely absolutely continuous with local multiplicity one almost everywhere on $\left(\frac{1}{2} ;+\infty\right)$ and pure point on $\left(-\infty ; \frac{1}{2}\right)$,

(d) If $\left|\frac{c_{1}^{2}+c_{2}^{2}-1}{c_{1} c_{2}}\right|>2$, the spectrum is pure point.

The four cases described above are illustrated by fig. 1 .

Proof. Without loss of generality, assume that $c_{1}, c_{2}>0$. Changing the sign of $c_{1}$ or $c_{2}$ leads to an unitarily equivalent operator.

Consider subordinacy properties of generalized eigenvectors [12].

If $\frac{\left|c_{1}^{2}+c_{2}^{2}-1\right|}{c_{1} c_{2}}>2$, we have $\left|\alpha_{-}\right|<1<\left|\alpha_{+}\right|$. By Lemma 2.2, $u_{-}$is a subordinate solution and lies in $l^{2}(\mathbb{N})$. Thus, every real $\lambda$ can either be an eigenvalue or belong to the resolvent set of the operator $J$.

If $\frac{\left|c_{1}^{2}+c_{2}^{2}-1\right|}{c_{1} c_{2}}<2$, we have $\operatorname{Re} \alpha_{+}=\operatorname{Re} \alpha_{-}, \operatorname{Re} \beta_{+}=\operatorname{Re} \beta_{-},\left|u_{n}^{+}\right| \sim\left|u_{n}^{-}\right|$as $n \rightarrow \infty$, and there is no subordinate solution for all real $\lambda$. The spectrum of $J$ in this situation is purely absolutely continuous.

If $\frac{c_{1}^{2}+c_{2}^{2}-1}{c_{1} c_{2}}=2$, which is equivalent to $\left|c_{1}-c_{2}\right|=1$, then either $\lambda>\frac{1}{2}$ or $\lambda<\frac{1}{2}$. If $\lambda>\frac{1}{2}$, then $|\alpha|=1, \delta_{+}=-\delta_{-}>0$ and $u_{-}$is subordinate and lies in $l^{2}(\mathbb{N})$, hence $\lambda$ can either be an eigenvalue or belong to the resolvent set. If $\lambda<\frac{1}{2}$, then $|\alpha|=1$, both $\delta_{+}$and $\delta_{-}$are pure imaginary, $\left|u_{n}^{+}\right| \sim\left|u_{n}^{-}\right|$as $n \rightarrow \infty$, no subordinate solution exists and ultimately $\lambda$ belongs to purely absolutely continuous spectrum.

If $\frac{c_{1}^{2}+c_{2}^{2}-1}{c_{1} c_{2}}=-2$, which is equivalent to $c_{1}+c_{2}=1$, the subcases $\lambda>\frac{1}{2}$ and $\lambda<\frac{1}{2}$ change places, which completes the proof. 


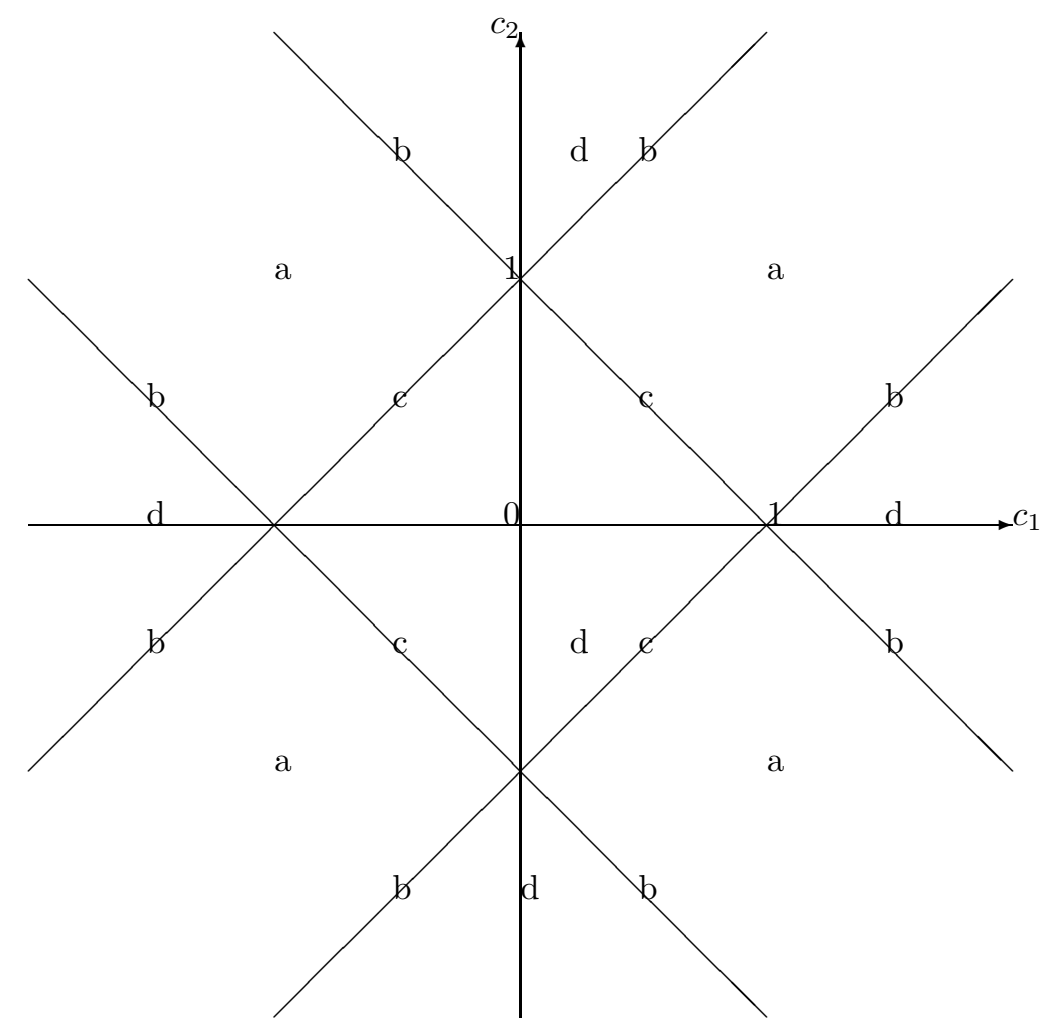

These results elaborate the domain structure, described in Section 1: we have obtained the information on the spectral structure of the operator $J$ when the modulation parameters are on the boundaries of regions.

\section{Criterion of SEmiboundedness And Discreteness of the SPECTRum}

We start with the following Theorem which constitutes a criterion of semiboundedness of the operator $J$.

Theorem 3.1. Let $c_{1} c_{2} \neq 0$.

1. If $\left|c_{1}\right|+\left|c_{2}\right|>1$, then the operator $J$ is not semibounded.

2. If $\left|c_{1}\right|+\left|c_{2}\right| \leq 1$, then the operator $J$ is semibounded from below.

Proof. Due to Theorem 2.3, there are four distinct cases of the spectral structure of the operator $J$, depending on the values of parameters $c_{1}$ and $c_{2}$ (see fig. 1).

The case (a), i.e., $\left|\frac{c_{1}^{2}+c_{2}^{2}-1}{c_{1} c_{2}}\right|<2$, is trivial, since $\sigma_{a c}(J)=\mathbb{R}$.

We are going to prove the assertion in the case (d), i.e., $\left|\frac{c_{1}^{2}+c_{2}^{2}-1}{c_{1} c_{2}}\right|>2$, using the result of Janas and Naboko 8. According to them, semiboundedness of the operator $J$ depends on the location of the point zero relative to the spectrum of the periodic operator $J_{\text {per }}$ ([8], see also Section 1$)$.

It is easy to see, that the absolutely continuous spectrum of the operator $J_{p e r}$ in our case consists of two intervals,

$$
\sigma_{a c}\left(J_{p e r}\right)=\left[\lambda_{-+} ; \lambda_{--}\right] \bigcup\left[\lambda_{+-} ; \lambda_{++}\right],
$$




$$
\begin{gathered}
\text { where } \lambda_{ \pm+}=1 \pm\left(\left|c_{1}\right|+\left|c_{2}\right|\right), \lambda_{ \pm-}=1 \pm|| c_{1}|-| c_{2}|| \text { and } \\
\lambda_{-+}<\lambda_{--}<1<\lambda_{+-}<\lambda_{++} .
\end{gathered}
$$

As it was established in $[8$, if the point zero lies in the gap between the intervals of the absolutely continuous spectrum of the operator $J_{\text {per }}$, then the operator $J$ is not semibounded. If, on the other hand, the point zero lies to the left of the spectrum of the operator $J_{\text {per }}$, then the operator $J$ is semibounded from below. A direct application of this result completes the proof in the case (d).

We now pass over to the cases (b) and (c), i.e., $\left|\frac{c_{1}^{2}+c_{2}^{2}-1}{c_{1} c_{2}}\right|=2, c_{1} c_{2} \neq 0$. This situation is considerably more complicated, since the point zero lies right on the edge of the absolutely continuous spectrum of the operator $J_{p e r}$. We consider the cases (b) and (c) separately.

(b):

We have to prove, that the operator $J$ is not semibounded. By Theorem 2.3 , $\sigma_{a c}(J)=\left(-\infty ; \frac{1}{2}\right]$, thus the operator $J$ is not semibounded from below. Now consider the quadratic form of the operator, taken on the canonical basis element $e_{n}$. We have

$$
\left(J e_{n}, e_{n}\right)=q_{n} \rightarrow+\infty, n \rightarrow \infty,
$$

thus the operator $J$ is not semibounded.

(c):

We will show that the operator $J$ is semibounded from below. To this end, we estimate its quadratic form: for any $u \in D(J)(D(J)$ being the domain of the operator $J$ ) one has

$$
\begin{aligned}
(J u, u)=\sum_{n=1}^{\infty} n\left|u_{n}\right|^{2}+\sum_{k=1}^{\infty} c_{1} & (2 k-1)\left(u_{2 k-1} \overline{u_{2 k}}+\right. \\
& \left.+\overline{u_{2 k-1}} u_{2 k}\right)+\sum_{k=1}^{\infty} c_{2}(2 k)\left(u_{2 k} \overline{u_{2 k+1}}+\overline{u_{2 k}} u_{2 k+1}\right) .
\end{aligned}
$$

Using the Cauchy inequality [1] and taking into account, that $\left|c_{1}\right|+\left|c_{2}\right|=1$, we ultimately arrive at the estimate

$$
\begin{gathered}
(J u, u) \geq \sum_{n=1}^{\infty} n\left|u_{n}\right|^{2}-\sum_{k=1}^{\infty}\left(\left|c_{1}\right|(2 k-1)\left|u_{2 k-1}\right|^{2}+\left|c_{1}\right|(2 k-1)\left|u_{2 k}\right|^{2}\right)- \\
-\sum_{k=1}^{\infty}\left(\left|c_{2}\right|(2 k)\left|u_{2 k}\right|^{2}+\left|c_{2}\right|(2 k)\left|u_{2 k+1}\right|^{2}\right)= \\
=\sum_{k=1}^{\infty}\left(\left|c_{1}\right|\left|u_{2 k}\right|^{2}+\left|c_{2}\right|\left|u_{2 k-1}\right|^{2}\right) \geq \min \left\{\left|c_{1}\right|,\left|c_{2}\right|\right\}\|u\|^{2}>0
\end{gathered}
$$

which completes the proof.

The remainder of the present Section is devoted to the proof of discreteness of the operator's pure point spectrum in the case (c) of Theorem 2.3. i.e., when $\left|c_{1}\right|+\left|c_{2}\right|=1, c_{1} c_{2} \neq 0$.

By Theorem 2.3, in this situation the absolutely continuous spectrum covers the interval $\left[\frac{1}{2} ;+\infty\right)$ and the remaining part of the spectrum, if it is present, is of pure point type. The estimate (3.1) obtained in the proof of the previous Theorem 
implies that there is no spectrum in the interval $\left(-\infty ; \min \left\{\left|c_{1}\right|,\left|c_{2}\right|\right\}\right)$. We will prove that nonetheless if $\left|c_{1}\right| \neq\left|c_{2}\right|$, the pure point spectral component of the operator $J$ is non-empty.

It is clear, that if $\left|c_{1}\right|=\left|c_{2}\right|=\frac{1}{2}$, the spectrum of the operator $J$ in the interval $\left(-\infty ; \frac{1}{2}\right)$ is empty and the spectrum in the interval $\left(\frac{1}{2} ;+\infty\right)$ is purely absolutely continuous. This situation together with its generalization towards Jacobi matrices with zero row sums was considered by Dombrowski and Pedersen in [3] and absolute continuity of the spectrum was established.

Theorem 3.2. In the case (c), i.e., when $\left|c_{1}\right|+\left|c_{2}\right|=1, c_{1} c_{2} \neq 0$, under an additional assumption $\left|c_{1}\right| \neq\left|c_{2}\right|$ the spectrum of the operator $J$ in the interval $\left(-\infty ; \frac{1}{2}\right)$ is non-empty.

Proof. Without loss of generality, assume that $0<c_{1}, c_{2}<1$. Changing the sign of $c_{1}, c_{2}$ or both leads to an unitarily equivalent operator.

Consider the quadratic form of the operator $J-\frac{1}{2} I$ for $u \in D(J)$.

$$
\begin{aligned}
\left(\left(J-\frac{1}{2} I\right) u, u\right) & =\sum_{n=1}^{\infty}\left[q_{n}\left|u_{n}\right|^{2}+\lambda_{n}\left(u_{n} \overline{u_{n+1}}+\overline{u_{n}} u_{n+1}\right)-\frac{1}{2}\left|u_{n}\right|^{2}\right]= \\
& =\sum_{n=1}^{\infty}\left[n\left|u_{n}\right|^{2}+c_{n} n\left(\left|u_{n+1}+u_{n}\right|^{2}-\left|u_{n+1}\right|^{2}-\left|u_{n}\right|^{2}\right)-\frac{1}{2}\left|u_{n}\right|^{2}\right] .
\end{aligned}
$$

Shifting the index $n$ by 1 in the term $c_{n} n\left|u_{n+1}\right|^{2}$ and then using the 2-periodicity of the sequence $\left\{c_{n}\right\}$, we have

$$
\begin{gathered}
\left(\left(J-\frac{1}{2} I\right) u, u\right)= \\
=\sum_{n=1}^{\infty}\left[c_{n} n\left|u_{n+1}+u_{n}\right|^{2}\right]+\sum_{n=1}^{\infty}\left[n\left|u_{n}\right|^{2}-c_{n} n\left|u_{n}\right|^{2}-c_{n+1}(n-1)\left|u_{n}\right|^{2}-\frac{1}{2}\left|u_{n}\right|^{2}\right]= \\
=\sum_{n=1}^{\infty}\left[c_{n} n\left|u_{n+1}+u_{n}\right|^{2}\right]+\sum_{n=1}^{\infty}\left(\frac{c_{n+1}-c_{n}}{2}\right)\left|u_{n}\right|^{2}= \\
=\sum_{k=1}^{\infty}\left[c_{1}(2 k-1)\left|u_{2 k-1}+u_{2 k}\right|^{2}+c_{2}(2 k)\left|u_{2 k}+u_{2 k+1}\right|^{2}\right]- \\
\quad-\left(\frac{c_{1}-c_{2}}{2}\right) \sum_{k=1}^{\infty}\left[\left|u_{2 k-1}\right|^{2}-\left|u_{2 k}\right|^{2}\right] .
\end{gathered}
$$

We need to find a vector $u \in D(J)$ which makes this expression negative. The following Lemma gives a positive answer to this problem via an explicit construction and thus completes the proof.

Lemma 3.3. For $0<c_{1}, c_{2}<1, c_{1}+c_{2}=1$ there exists a vector $u \in l_{\text {fin }}(\mathbb{N})$ such that

$$
\begin{aligned}
\sum_{k=1}^{\infty}\left[c_{1}(2 k-1)\left|u_{2 k-1}+u_{2 k}\right|^{2}+c_{2}(2 k) \mid\right. & \left.u_{2 k}+\left.u_{2 k+1}\right|^{2}\right]< \\
& <\left(\frac{c_{1}-c_{2}}{2}\right) \sum_{k=1}^{\infty}\left[\left|u_{2 k-1}\right|^{2}-\left|u_{2 k}\right|^{2}\right]
\end{aligned}
$$


Proof. We consider the cases $c_{1}>c_{2}$ and $c_{1}<c_{2}$ separately. Below we will see, that the latter can be reduced to the former.

1. $c_{1}-c_{2}>0$.

In this case, we will choose a vector $v$ from $l_{\text {fin }}(\mathbb{N})$ with nonnegative components such that if the vector $u$ is defined by $u_{2 k-1}=v_{k}, u_{2 k}=-v_{k+1}$, the condition (3.2) holds true. In terms of such $v$, the named condition admits the following form:

$$
c_{1} \sum_{k=1}^{\infty}\left[(2 k-1)\left(v_{k}-v_{k+1}\right)^{2}\right]<\left(\frac{c_{1}-c_{2}}{2}\right) v_{1}^{2}
$$

2. $c_{1}-c_{2}<0$.

In this case, we will choose a vector $w \in l_{\text {fin }}(\mathbb{N})$ with nonnegative components and the value $t$ such that if the vector $u$ is defined by $u_{2 k}=-w_{k}, u_{2 k-1}=w_{k}, u_{1}=$ $t w_{1}$, the condition (3.2) holds true. In terms of $w$ and $t$, condition (3.2) admits the form

$$
c_{2} \sum_{k=1}^{\infty}\left[(2 k)\left(w_{k}-w_{k+1}\right)^{2}\right]<\left(-\frac{t^{2}}{2}+2 c_{1} t+\frac{1-4 c_{1}}{2}\right) w_{1}^{2}
$$

Take $t$ such that the expression in the brackets on the right-hand side of the latter inequality is positive. This choice is possible, if we take take the maximum of the parabola $y(t)=-\frac{t^{2}}{2}+2 c_{1} t+\frac{1-4 c_{1}}{2}$, located at the point $t_{0}=2 c_{1}$. Then the inequality (3.4) admits the form

$$
c_{2} \sum_{k=1}^{\infty}\left[(2 k)\left(w_{k}-w_{k+1}\right)^{2}\right]<\frac{\left(c_{1}-c_{2}\right)^{2}}{2} w_{1}^{2}
$$

We will now explicitly construct a vector $v^{(N)} \in l_{\text {fin }}(\mathbb{N})$ such that it satisfies both (3.3) and (3.5) for sufficiently large numbers of $N$. Consider the sequence $v_{n}^{(N)}=\sum_{k=n}^{N} \frac{1}{k}$ for $n \leq N$ and put $v_{n}^{(N)}=0$ for $n>N$. It is clear, that as $N \rightarrow+\infty$,

and

$$
\left(v_{1}^{(N)}\right)^{2}=\left(\sum_{k=1}^{N} \frac{1}{k}\right)^{2} \sim(\ln N)^{2},
$$

$$
\sum_{k=1}^{\infty}\left[(2 k-1)\left(v_{k}^{(N)}-v_{k+1}^{(N)}\right)^{2}\right] \sim \sum_{k=1}^{\infty}\left[(2 k)\left(v_{k}^{(N)}-v_{k+1}^{(N)}\right)^{2}\right] \sim 2 \ln N=o\left(\left(v_{1}^{(N)}\right)^{2}\right),
$$

which completes the proof of Lemma 3.3

We are now able to prove the discreteness of the pure point spectral component of the operator $J$ in the case (c) of Theorem 2.3, which is non-empty due to Theorem 3.2 .

Theorem 3.4. In the case (c), i.e., when $\left|c_{1}\right|+\left|c_{2}\right|=1$ and $c_{1} c_{2} \neq 0$, under an additional assumption $\left|c_{1}\right| \neq\left|c_{2}\right|$ the spectrum of the operator $J$ in the interval $\left(-\infty ; \min \left\{\left|c_{1}\right|,\left|c_{2}\right|\right\}\right)$ is empty, the spectrum in the interval $\left[\min \left\{\left|c_{1}\right|,\left|c_{2}\right|\right\} ; \frac{1}{2}\right)$ is discrete, and the following estimate holds for the number of eigenvalues $\lambda_{n}$ in the interval $\left(-\infty ; \frac{1}{2}-\varepsilon\right), \varepsilon>0$ :

$$
\#\left\{\lambda_{n}: \lambda_{n}<\frac{1}{2}-\varepsilon\right\} \leq \frac{1}{\varepsilon} .
$$


Proof. According to the Glazman Lemma 1, dimension of the spectral subspace, corresponding to the interval $\left(-\infty ; \frac{1}{2}-\varepsilon\right)$, is less or equal to the co-dimension of any subspace $H_{\varepsilon} \subset l^{2}(\mathbb{N})$ such that

$$
(J u, u) \geq\left(\frac{1}{2}-\varepsilon\right)\|u\|^{2}
$$

for any $u \in D(J) \bigcap H_{\varepsilon}$. Consider subspaces $l_{N}^{2}$ of vectors with zero first $N$ components, i.e., $l_{N}^{2}:=\left\{u \in l^{2}(\mathbb{N}): u_{1}=u_{2}=\cdots=u_{N}=0\right\}$. For any $\varepsilon, 0<\varepsilon<\frac{1}{2}$ we will find a number $N(\varepsilon)$ such that for any vector from $H_{\varepsilon}=l_{N(\varepsilon)}^{2}$ the inequality (3.6) is satisfied. We consider $\varepsilon$ such that $0<\varepsilon<\frac{1}{2}$ only, since the spectrum is empty in the interval $(-\infty ; 0]$ (see the estimate (3.1)). The co-dimension of the subspace $l_{N(\varepsilon)}^{2}$ is $N(\varepsilon)$, so this value estimates from above the number of eigenvalues in the interval $\left(-\infty ; \frac{1}{2}-\varepsilon\right)$.

Consider the quadratic form of the operator $J$ for $u \in D(J)$ :

$$
\begin{gathered}
(J u, u)=\sum_{n=1}^{\infty} n\left[\left|u_{n}\right|^{2}+c_{n} 2 \operatorname{Re}\left(u_{n} \overline{u_{n+1}}\right)\right] \geq \\
\geq \sum_{n=1}^{\infty} n\left[\left|u_{n}\right|^{2}-\left|c_{n}\right|\left(I_{n}\left|u_{n}\right|^{2}+\frac{1}{I_{n}}\left|u_{n+1}\right|^{2}\right)\right]= \\
=\left|u_{1}\right|^{2}\left(1-\left|c_{1}\right| I_{1}\right)+\sum_{n=2}^{\infty} n\left|u_{n}\right|^{2}\left[1-\left|c_{n}\right| I_{n}-\left|c_{n-1}\right| \frac{1}{I_{n-1}} \frac{n-1}{n}\right],
\end{gathered}
$$

where we have used the Cauchy inequality [1] $2 R e\left(u_{n} \overline{u_{n+1}}\right) \leq I_{n}\left|u_{n}\right|^{2}+\frac{1}{I_{n}}\left|u_{n+1}\right|^{2}$ with the sequence $I_{n}>0, n \in \mathbb{N}$ which we will fix as $I_{n}:=1-\frac{\phi}{n}$ in order that the expression

$$
1-\left|c_{n}\right| I_{n}-\left|c_{n-1}\right| \frac{1}{I_{n-1}}\left(1-\frac{1}{n}\right)
$$

takes its simplest form. This choice cancels out the first order with respect to $n$. The value of $\phi$ in the interval $0<\phi<1$ will be fixed later on. We have:

$$
\frac{1}{I_{n-1}}=1+\frac{\phi}{n}+\phi_{n}
$$

where $\phi_{n}=O\left(\frac{1}{n^{2}}\right), n \rightarrow \infty$. Moreover, as can be easily seen,

$$
\phi_{n}=\frac{\phi(\phi+1)}{n(n-1-\phi)} \text {. }
$$

After substituting the value of $\phi_{n}$ into (3.8) and then into (3.7) we obtain:

$$
1-\left|c_{n}\right| I_{n}-\left|c_{n-1}\right| \frac{1}{I_{n-1}}\left(1-\frac{1}{n}\right)=\frac{1}{n}\left(\phi\left|c_{n}\right|+(1-\phi)\left|c_{n-1}\right|\right)+\theta_{n}
$$

with $\theta_{n}:=\left|c_{n-1}\right|\left(\frac{\phi}{n^{2}}-\phi_{n}\left(1-\frac{1}{n}\right)\right)=O\left(\frac{1}{n^{2}}\right)$ as $n \rightarrow \infty$.

Choose $\phi$ in order to make the right hand side of expression (3.9) symmetric with respect to the modulation parameters $c_{1}$ and $c_{2}: \phi=\frac{1}{2}$. Then

$$
1-\left|c_{n}\right| I_{n}-\left|c_{n-1}\right| \frac{1}{I_{n-1}}\left(1-\frac{1}{n}\right)=\frac{1}{2 n}+\theta_{n} .
$$


Consequently,

$$
(J u, u) \geq\left|u_{1}\right|^{2}\left(1-\frac{\left|c_{1}\right|}{2}\right)+\sum_{n=2}^{\infty}\left(\frac{1}{2}+\theta_{n} n\right)\left|u_{n}\right|^{2} .
$$

Since $n \theta_{n} \rightarrow 0$ as $n \rightarrow \infty$, we can choose $N(\varepsilon)$ such that for any $n>N(\varepsilon)$ the condition $n \theta_{n}>-\varepsilon$ holds. Thus condition (3.6) will be satisfied for all vectors from $D(J) \cap l_{N(\varepsilon)}^{2}$, since their first components are zeros.

The discreteness of the pure point spectrum is proved. We pass on to the proof of the estimate for $N(\varepsilon)$. We start with $\theta_{n}$ :

We have

$$
\left|\theta_{n}\right| \leq\left|\frac{\phi}{n^{2}}-\frac{\phi(\phi+1)}{n(n-1-\phi)} \frac{n-1}{n}\right|=\frac{1}{2 n^{2}}\left|1-\frac{3}{2} \frac{n-1}{n-\frac{3}{2}}\right| .
$$

$$
n>2 \Rightarrow\left\{2>\frac{n-1}{n-\frac{3}{2}}>1\right\} \Rightarrow\left\{n\left|\theta_{n}\right|<\frac{1}{4 n}\right\} .
$$

Taking $N(\varepsilon)=\frac{1}{\varepsilon}, 0<\varepsilon<\frac{1}{2}$, we see that for any $n>N(\varepsilon)>2$ the condition $n \theta_{n}>$ $-\varepsilon$ holds. Thus, the condition (3.6) is satisfied for all vectors from $D(J) \cap l_{N(\varepsilon)}^{2}$, which completes the proof.

\section{The Degenerate CASE}

Now we consider the case, when one of the modulation parameters turns to zero (we call this case degenerate). Formally speaking, we cannot call such matrix a Jacobi one, but this limit case is of certain interest for us, supplementing the whole picture.

Theorem 4.1. If $c_{1} c_{2}=0, c \neq 0$ (denoting $\left.c:=\max \left\{\left|c_{1}\right|,\left|c_{2}\right|\right\}\right)$, then the spectrum of the operator $J$ is the closure of the set of eigenvalues $\lambda_{n}$ :

$$
\sigma(J)=\overline{\left\{\lambda_{n}, n \in \mathbb{N}\right\}} .
$$

The set of eigenvalues is

$$
\left\{\lambda_{n}, n \in \mathbb{N}\right\}=\left\{\begin{array}{l}
\left\{\lambda_{n}^{+}, \lambda_{n}^{-}, n \in \mathbb{N}\right\}, \text { if } c_{1} \neq 0, c_{2}=0 \\
\left\{1, \tilde{\lambda}_{n}^{+}, \tilde{\lambda}_{n}^{-}, n \in \mathbb{N}\right\}, \text { if } c_{1}=0, c_{2} \neq 0,
\end{array}\right.
$$

where eigenvalues $\lambda_{n}^{ \pm}, \tilde{\lambda}_{n}^{ \pm}$have the following asymptotics:

$$
\begin{gathered}
\lambda_{n}^{+}, \tilde{\lambda}_{n}^{+}=2(1+c) n+O(1), n \rightarrow \infty, \\
\lambda_{n}^{-}=2(1-c) n+\left(c-\frac{1}{2}\right)-\frac{1}{16 c n}+O\left(\frac{1}{n^{2}}\right), n \rightarrow \infty \\
\tilde{\lambda}_{n}^{-}=2(1-c) n+\left(2 c-\frac{3}{2}\right)-\frac{1}{16 c n}+O\left(\frac{1}{n^{3}}\right), n \rightarrow \infty,
\end{gathered}
$$

Proof. When one of the parameters $c_{1}$ or $c_{2}$ is zero, the infinite matrix consists of $2 \times 2$ (or $1 \times 1$ ) blocks. Thus, the operator $J$ is an orthogonal sum of finite $2 \times 2$ (or 1x1) matrices $J_{n}, J=\bigoplus_{n=1}^{\infty} J_{n}$. Then, the spectrum of the operator $J$ is the closure of the sum of spectrums of these matrices, $\sigma(J)=\overline{\bigcup_{n=1}^{\infty} \sigma\left(J_{n}\right)}$. Let us calculate $\sigma\left(J_{n}\right)$.

If $c_{1} \neq 0, c_{2}=0$, then

$$
J_{n}=\left(\begin{array}{cc}
2 n-1 & c_{1}(2 n-1) \\
c_{1}(2 n-1) & 2 n
\end{array}\right)
$$


and $\sigma\left(J_{n}\right)=\left\{\lambda_{n}^{+}, \lambda_{n}^{-}\right\}$, where $\lambda_{n}^{ \pm}=\frac{4 n-1 \pm \sqrt{4 c^{2}(2 n-1)^{2}+1}}{2}$ and it is easy to see that

$$
\lambda_{n}^{ \pm}=2(1 \pm c) n-\left(\frac{1}{2} \pm c\right) \pm \frac{1}{16 c n}+O\left(\frac{1}{n^{2}}\right), n \rightarrow \infty .
$$

If $c_{1}=0, c_{2} \neq 0$, then $J_{1}=1, \sigma\left(J_{1}\right)=\{1\}$,

$$
J_{n}=\left(\begin{array}{cc}
2 n-2 & c_{2}(2 n-2) \\
c_{2}(2 n-2) & 2 n-1
\end{array}\right), n \geq 2
$$

and $\sigma\left(J_{n}\right)=\left\{\tilde{\lambda}_{n}^{+}, \tilde{\lambda}_{n}^{-}\right\}, n \geq 1$, where $\tilde{\lambda}_{n}^{ \pm}=\frac{4 n-3 \pm \sqrt{4 c^{2}(2 n-2)^{2}+1}}{2}$ and it is easy to see that

$$
\tilde{\lambda}_{n}^{ \pm}=2(1 \pm c) n-\left(\frac{3}{2} \pm 2 c\right) \pm \frac{1}{16 c n}+O\left(\frac{1}{n^{2}}\right), n \rightarrow \infty,
$$

which completes the proof.

Remark 4.2. From the last Theorem it follows that as $n \rightarrow \infty, \lambda_{n}^{+}, \tilde{\lambda}_{n}^{+} \rightarrow+\infty$. As for $\lambda_{n}^{-}$and $\tilde{\lambda}_{n}^{-}$, their asymptotic behavior depends on the parameter $c$ :

If $c>1$, then $\lambda_{n}^{-}, \tilde{\lambda}_{n}^{-} \rightarrow-\infty$.

If $c=1$, then $\lambda_{n}^{-}, \tilde{\lambda}_{n}^{-} \rightarrow \frac{1}{2}$.

Finally, if $0<c<1$, then $\lambda_{n}^{-}, \tilde{\lambda}_{n}^{-} \rightarrow+\infty$.

Hence, if $0<c \leq 1$, the operator $J$ is semibounded from below, and if $c>1$, the operator $J$ is not semibounded. This clearly corresponds to results, obtained in Section 3.

When we move along the side of the boundary square (see fig. 1, case (c)) towards one of the points $\left\{D_{j}\right\}_{j=1}^{4}=\{(1 ; 0) ;(0 ; 1) ;(-1 ; 0) ;(0 ;-1)\}$, the absolutely continuous spectrum covers the interval $\left[\frac{1}{2} ;+\infty\right)$. At the same time, at each limit point $D_{j}, j=1,2,3,4$, the spectrum of $J$ becomes pure point, which demonstrates yet another phenomenon of the spectral phase transition. Moreover, note that the spectrum at each limit point consists of two series of eigenvalues, one going to $+\infty$, another accumulating to the point $\lambda=\frac{1}{2}$, both points prior to the spectral phase transition having been the boundaries of the absolutely continuous spectrum.

Remark 4.3. The proof of discreteness of the spectrum in the case (c) of Theorem 2.3 essentially involves the semiboundedness property of the operator $J$. In the case (b) one does not have the advantage of semiboundedness and due to that reason the proof of discreteness supposedly becomes much more complicated.

Remark 4.4. The choice $q_{n}=n$ was determined by the possibility to apply the Birkhoff-Adams technique. It should be mentioned that much more general situation $q_{n}=n^{\alpha}, 0<\alpha<1$ may be considered on the basis of the generalized discrete Levinson Theorem. Proper approach has been developed in [11, see also [5]. One can apply similar method in our situation. Another approach which is also valid is so-called Jordan box case and is presented in [7].

\section{ACKNOWLEDGEMENTS}

The author expresses his deep gratitude to Prof. S. N. Naboko for his constant attention to this work and for many fruitful discussions of the subject and also to Dr. A. V. Kiselev for his help in preparation of this paper. 


\section{REFERENCES}

[1] N. I. Akhiezer, I. M. Glazman, Theory of linear operators in Hilbert space. (2nd edition) Dover, New York, 1993.

[2] Yu. M. Berezanskii, Expansions in eigenfunctions of selfadjoint operators. (Russian) Naukova Dumka, Kiev, 1965.

[3] J. Dombrowski, S. Pedersen, Absolute continuity for unbounded Jacobi matrices with constant row sums. J. Math. Anal. Appl., vol. 267(2002), no. 2, pp. 695-713.

[4] S. N. Elaydi, An Introduction to Difference Equations. Springer-Verlag, New York, 1999.

[5] D. Damanik, S. N. Naboko, A first order phase transition in a class of unbounded Jacobi matrices: critical coupling. (to appear in Journal Approximation Theory)

[6] D. Gilbert, D. Pearson, On subordinacy and analysis of the spectrum of one dimensional Schrödinger operators. J. Math. Anal. Appl., vol. 128 (1987), pp. 30-56.

[7] J. Janas, The asymptotic analysis of generalized eigenvectors of some Jacobi operators. Jordan box case. (to appear in J. Difference Eq. Appl.)

[8] J. Janas, S. N. Naboko, Criteria for semiboundedness in a class of unbounded Jacobi operators. translation in St. Petersburg Math. J., vol. 14 (2003), no. 3, pp. 479-485.

[9] J. Janas, S. N. Naboko, Multithreshold spectral phase transition examples for a class of unbounded Jacobi matrices. Oper. Theory Adv. Appl., vol. 124 (2001), pp. 267-285.

[10] J. Janas, S. N. Naboko, Spectral analysis of selfadjoint Jacobi matrices with periodically modulated entries. J. Funct. Anal., vol. 191 (2002), no. 2 , pp. 318-342.

[11] J. Janas, S. N. Naboko, E. Sheronova, Asymptotic behaviour of generalizes eigenvectors of Jacobi matrices in Jordan box case. (submitted to Rocky Mount. Math. J.)

[12] S. Khan, D. Pearson, Subordinacy and spectral theory for infinite matrices. Helv. Phys. Acta, vol. 65 (1992), pp. 505-527.

Department of Mathematical Physics, Institute of Physics, St. Petersburg University, Ulianovskaia 1, 198904, St. Petergoff, St. Petersburg, Russia

E-mail address: sergey_simonov@mail.ru 